\title{
QUALITY IMPROVEMENT Increasing advance care planning in the secondary care setting: A quality improvement project
}

\author{
Authors: Anna Steel ${ }^{A}$ and Deborah Bertfield ${ }^{B}$
}

\section{Background}

Advance care plans (ACP) provide patients the opportunity to communicate their goals and wishes for future care.

\section{Local problem}

A retrospective case note review of 50 inpatient deaths in 2017 confirmed a doctor had discussed expected death in $90 \%$, however only $2 \%$ had an ACP.

\section{Methods}

Patients appropriate for ACP were identified on a single geriatrics ward. Interventions were implemented with monthly data collection. Patients with an ACP were followed prospectively. The initiatives were subsequently applied across six geriatrics wards.

\section{Interventions}

Interventions included improved identification of patients appropriate for ACP, doctor education and improved communication to general practitioners and healthcare providers.

Results

Before initiation of interventions on the pilot ward, ACP was completed for $38 \%$ of appropriate patients; this increased to a mean of $78.6 \%$ over 4 months post-interventions. During the pilot, 44 patients had an ACP. Of those discharged, $75 \%$ avoided readmission over the following 6 months. After applying the interventions across all geriatric wards, ACPs increased to a mean of $81.2 \%$ and was maintained 12 months later at $72 \%$.

\section{Conclusions}

The initiatives formed a structure to promote the use of ACP on the wards. Care plans focused on individualising care and effective communication resulted in reduction of readmissions.

KEYWORDS: Advance care planning, end-of-life, ACP

DOI: 10.7861/fhj.2019-0040

\section{Introduction}

The nature of the problem

The Gold Standards Framework defines advance care planning (ACP) as the "conversation between people, their families and

Authors: ${ }^{A}$ geriatrics registrar, Barnet Hospital, Barnet, UK; ${ }^{B}$ consultant geriatrician, Barnet Hospital, Barnet, UK carers and those looking after them about their future wishes and priorities for care.'. In 2018, The Royal College of Physicians (RCP) published key recommendations that included to 'initiate shared decision-making including ACP in line with patient preferences'.

$\mathrm{ACP}$ is an ongoing process through which a patient can express their preferences for future health and end-of-life care. In an era when medical interventions can prolong life even beyond what a person may wish, an ACP can help navigate through these difficult medical decisions so that the patient, rather than their condition, is treated. ${ }^{3,4}$ If a patient lacks capacity, a care plan can be generated with a designated proxy decision-maker acting in their best interests.

A retrospective review of deaths in the hospital was undertaken to determine the frequency with which an ACP was discussed. These data confirmed that significant improvement was required to ensure more patients were provided the opportunity to receive an ACP and consequently a better end-of-life experience.

\section{The evidence for advance care planning}

The disease trajectory in chronic conditions is unpredictable and even more challenging in frail older people. ${ }^{5}$ Most patients do not want to die in hospital and do not enjoy the hospital experience. ${ }^{6}$ However, when patients are approaching the end of life, they have most contact with hospital and social services. ${ }^{7,8}$ Multiple transfers between care settings do little to improve quality of life or symptom control and may threaten continuity and safety. ${ }^{9} \mathrm{ACP}$, in conjunction with specialist palliative care, reduces hospitalisation, multiple readmissions and helps patients to die in their preferred location. $^{10}$

The RCP's Talking about dying report discusses when to initiate ACP discussions, suggesting that 'conversations about the future should be initiated at any point'. ${ }^{2}$ Patients state they want their doctor to have these discussions sooner rather than later. ${ }^{11,12}$ Others, however, may not feel ready or able to have this conversation and the timing of the discussion is likely to influence its acceptability and effect. ${ }^{13}$ When a patient is acutely unwell, making treatment decisions can be stressful and rushed. By planning for these eventualities in advance, it allows patients and their caregivers to feel prepared and more in control, improving their experience. ${ }^{14}$ Patients and families do not mind having these ACP discussions with someone other than their 'regular doctor' as long as the doctor is willing to open the conversation with them. ${ }^{15}$ Patients describe an enhanced doctor-patient relationship after discussing end-of-life plans. ${ }^{16}$ It is suggested that caution is required because ACPs can oversimplify the decision-making 
process if not followed up appropriately. Decisions will likely change over time due to social, cultural, financial and other circumstantial factors. ${ }^{17}$

At the end of life, there is often a disparity between the patient's expressed preference and what a physician or family member thinks they would choose. ${ }^{18}$ Contention can arise within families and with healthcare providers (HCPs) when the patient's preferences are unknown. ${ }^{19}$ Conducting an ACP discussion removes the uncertainty and fear which can result in conflict. In addition, the bereaved relatives of patients with ACPs feel less anxiety and guilt after the death. ${ }^{20}$

\section{Barriers to advance care planning}

Relatives and carers may adopt the 'not yet' approach and the need to 'stay positive.' ${ }^{4}$ Culturally, discussion and acceptance of death can be difficult. ${ }^{21}$ Patients and relatives may lack the confidence to initiate conversations about death and prognosis.

HCPs may struggle with discussing the end of life. Addressing their own acceptance of disease progression can be difficult as they may view it as a therapeutic failure. ${ }^{22}$ It can be difficult to know when to initiate conversations and HCPs may fear doing it too early. ${ }^{15}$ Many chronically ill patients are already thinking about their end-of-life decisions and would be willing to discuss them. ${ }^{23}$ Few doctors have had any formal training in having these discussions and many believe that they lack the appropriate skills, experience or knowledge to comfortably perform ACP. ${ }^{24,25}$ Time constraints for hospital physicians have also been identified as an important barrier. ${ }^{26}$

The project aims and rationale behind the interventions

Having identified a need to improve ACP for older people towards the end of life, key priorities for interventions were established. Considerable emphasis has been placed on leadership for the sustainability of successful interventional projects with ACP leads providing face-to-face training, mentoring, updates, networking and development of processes. ${ }^{26}$ The role of the ACP lead in this study was to coordinate the initiative to ensure a sustained improvement.

\section{Aims}

Improve recognition of patients who are appropriate for ACP

The time constraints that HCPs face in the hospital often result in ACP discussions being considered less important than daily routines with more measurable outcomes. ${ }^{27}$ An organisational commitment to ACP promotes implementation. ${ }^{26}$ Routinely highlighting patients requiring an ACP at the daily multidisciplinary meeting could help to prioritise and legitimise the task. Physicians acting as ACP champions have successfully promoted ACP discussions among colleagues. ${ }^{26}$ The 'ACP champions' role was designated to the ward registrars.

\section{Improve doctor education in ACP and end of life}

Education and communication programmes instil confidence in doctors to engage with early ACP conversations. ${ }^{28,29}$ To address this, a series of educational events were designed to increase doctors' confidence and ability to have effective ACP discussions with patients.

\section{Improve communication of ACP discussions between primary and secondary care}

Effective communication of information is essential to implement patients' choices in end-of-life decisions. ${ }^{30}$ The NHS Five year forward view recognises the collaboration and communication between HCPs. ${ }^{31}$ To ensure this, emphasis was placed on detailed written documentation and, on occasion, telephone calls to primary care providers.

\section{Methods}

For the quality improvement project (QIP), a plan, do, study, act (PDSA) cycle was used. To identify the nature of the problem and 'plan' the QIP, a pre-project audit was performed.

\section{Pre-project audit}

A retrospective case-note review of all hospital deaths over a 4 -week period was undertaken. To improve ACP, three key strategies were highlighted for implementation. Firstly, identification of patients appropriate for ACP discussion; secondly, doctor education; and finally, improved communication with primary care.

\section{Identifying appropriate patients for initiation of ACP discussion}

Triggers to consider which patients were appropriate for initiation of an ACP discussion included trust guidelines, a high admission clinical frailty score, consideration of the 'surprise question', a preexisting do not attempt cardiopulmonary resuscitation (DNA-CPR) form and patient choice. ${ }^{1,32}$ For patients who lacked capacity, care planning was discussed with the next of kin. A new ACP column on the patient whiteboard was created to prompt initiation of discussion of appropriate patients with further prompting from the designated 'ACP champion'.

\section{Doctor education}

Three lectures and multiple informal tutorials on ACP discussions were given to doctors within the geriatrics department. Posters detailing when and how to do ACP were created. To increase doctor confidence, junior team members shadowed seniors performing ACPS.

\section{Improved communication with primary care}

ACP stickers were printed for the medical notes highlighting documentation. Junior doctor education focused on improving communication with primary care on discharge summaries and verbally. Doctors reviewed the general practitioner (GP) summary care records and asked patients or their proxy decision-maker whether or not a prior ACP discussion had taken place. Prior to a patient's discharge, doctors were encouraged in their ACP training to telephone GPs communicating information from the ACP discussion. 


\section{Data collection}

Following the retrospective review, baseline data were collected on the pilot ward. The current patient notes and any corresponding online information were reviewed. Patients considered appropriate for an ACP and those who had an ACP already in place were identified.

Patients who had an ACP during the intervention period were prospectively followed up over 6 months. Hospital records were reviewed to determine if the patient had been readmitted or died and the location of death.

Interventions introduced across remaining five general geriatric wards

Once the pilot was established and the strategies had improved the number of patients with an ACP, the project expanded to include five other geriatric wards. Through studying the outcomes of the pilot as part of the PDSA cycle, it was noted that the team needed regular prompting at the board round to consider each patient for ACP. ACP champions were therefore recruited to the remaining five wards to ensure the interventions outlined occurred on each ward. Teaching sessions were conducted for all doctors in the geriatrics department.

Baseline data were collected for each of the five geriatric wards. Once the interventions were implemented, the percentage of patients who were appropriate for ACP and who had one initiated was recorded weekly over a 5 -week period. ACP champions received weekly reminders to ensure the boards were updated.

\section{2-month follow-up}

The project continued on all six wards over the subsequent year with the same interventions. When the junior doctors rotated, training was implemented and new ACP champions were recruited. At 12 months, data were recorded weekly for a 6 -week period.

There was no need for formal ethics review and no conflict of interest.

\section{Results}

\section{Pre-project audit}

There were 80 deaths in the hospital in September 2017. It was only possible to retrieve and review 50 of these case-notes.

The mean age of the patients was 86 years with a mean Rockwood clinical frailty score of 6.2. The mean duration from admission to death was 16.9 days.

Ninety-eight per cent of the patients had a DNA-CPR form and $90 \%$ of families had discussed the anticipated death with the doctors. One patient had a documented ACP. Applying the triggers discussed, ACP discussion would have been appropriate in $98 \%$ of the patients.

Thirty-four per cent of this cohort had a recent hospital admission (within a mean of 3 months from discharge to readmission).

\section{Identifying appropriate patients}

At baseline, 13 (59\%) patients on the pilot ward were considered appropriate to have an ACP discussion. Of these, ACP was initiated in five (38\%).

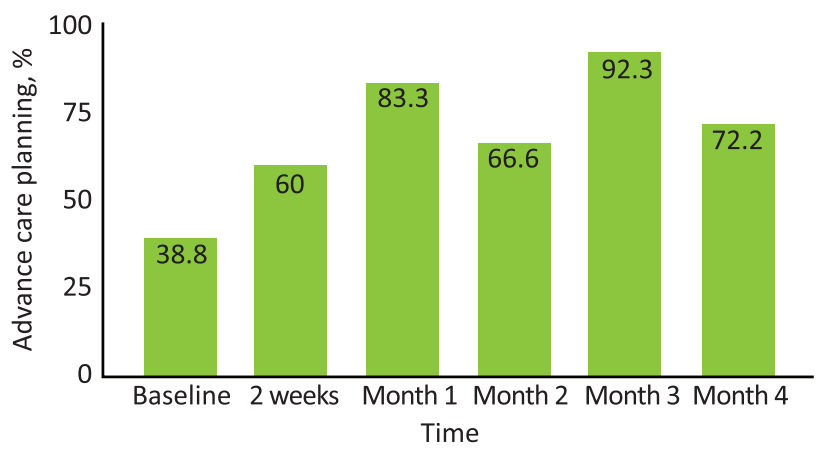

Fig 1. Advance care planning initiated post-intervention.

\section{Doctor education}

Following introduction of the ACP column and prompting from the ACP champions, there was an initial increase in ACPs completed to $60 \%$. Following targeted junior doctor education on the ward and with lectures, this increased to $83.3 \%$ (Fig 1).

\section{Improved communication with primary care}

The pilot study data showed that $93 \%$ of ACP discussions were communicated to primary care in the discharge summary.

\section{Data collection}

During the pilot study, a mean of $78.6 \%$ of patients had an ACP discussion initiated appropriately. The percentage of appropriate ACPs completed dropped to $20 \%$ when the ACP champion was absent but increased to $91 \%$ within 2 weeks of their return (Fig 1).

Over the 4-month period of the pilot, a total of 44 patients had an ACP; 20 (45\%) of these patients died during admission. Of the 24 (55\%) who were discharged, hospital records were monitored to see if they were readmitted or died. Eighteen (75\%) patients were not readmitted in accordance with their wishes. Of the patients discharged and not readmitted, 15 (83\%) died in their chosen location in the community. None of the patients chose hospital as their preferred place of death. Six patients were readmitted and their reasons are charted in Fig 2.

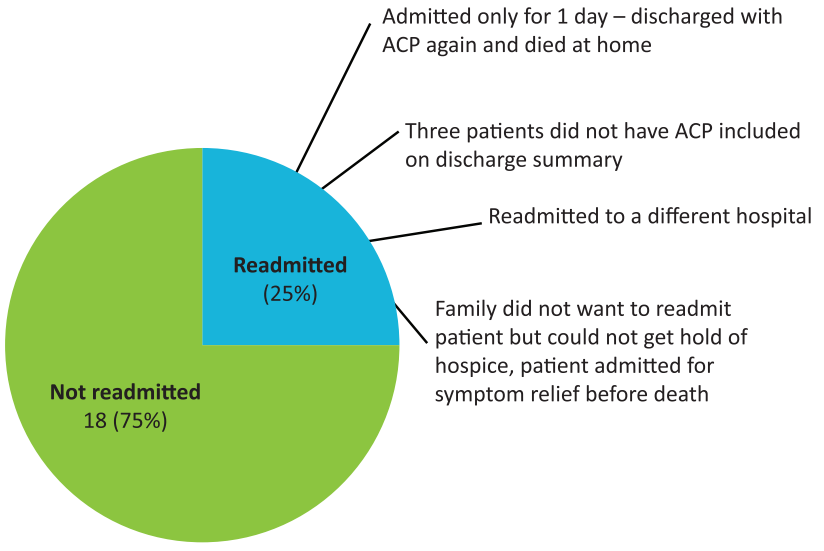

Fig 2. Patients readmitted post-advance care planning. $A C P=$ advance care planning. 


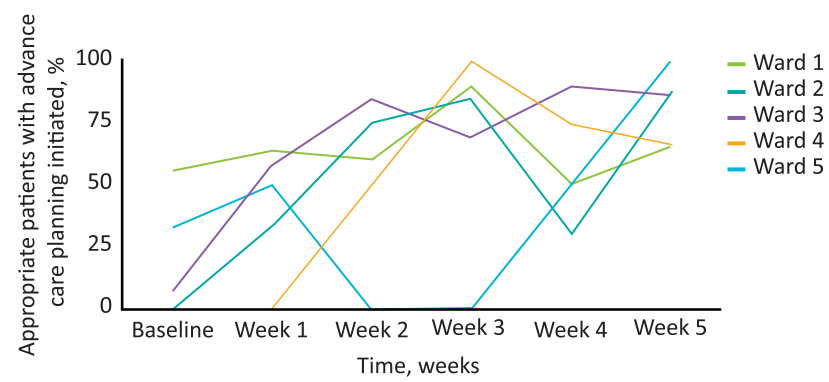

Fig 3. Appropriate patients with advance care planning initiated on five remaining general geriatric wards.

Interventions introduced across the remaining five geriatrics wards

At baseline before introduction of the interventions, $19.2 \%$ of patients considered appropriate had an ACP initiated. After 5 weeks of the project, the mean rate of ACPs initiated when appropriate was $81.2 \%$ (Fig 3).

\section{Follow-up at 12 months}

At 12 months, data were collected for each ward over 6 weeks. The mean rate of ACPs initiated across all six wards during this time period was $72.3 \%$ (range $55.3 \%$ to $90.6 \%$; Fig 4 ).

\section{Discussion}

The project demonstrates that through simple interventions, education, an ACP section on the board round and use of ACP champions, there was a marked increase in the number of ACPs initiated in appropriate patients. The ACP champion was key to the project's success and sustainability. When the ACP champion was absent, the number of ACPs reduced significantly but was restored when they returned. In the second month of the project, there was a reduction of ACPs completed when the junior doctors rotated (and before the new juniors received training).

There was improved communication with primary care, with $93 \%$ of ACP discussions included on the discharge summaries; $75 \%$ of patients discharged were not readmitted (in the time of the study) and, of these, $83 \%$ died in their preferred location.

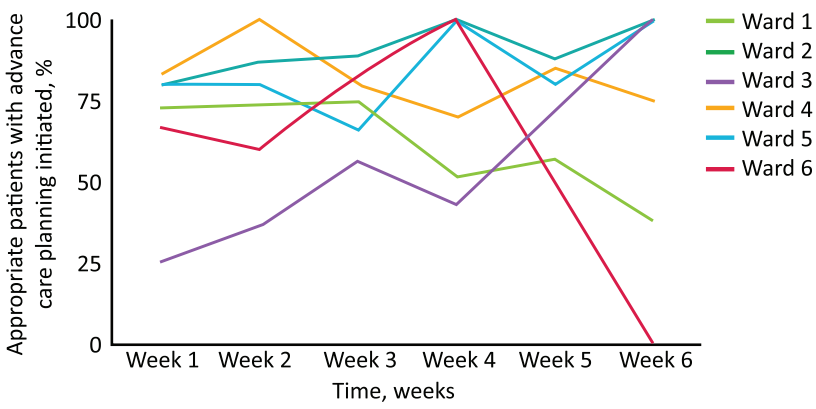

Fig 4. 12-month follow-up for appropriate patients with advance care planning initiated across all six general geriatric wards.
The project has increased initiation of ACPs across the whole department in appropriate patients with a sustained change in practice.

\section{Interpretation}

In the pre-project audit, $98 \%$ of patients who died would have been appropriate for an ACP discussion; $34 \%$ of these patients had a recent hospital admission with a mean time of 3 months suggesting that there may have been an opportunity on the previous admission for an ACP discussion.

There was a higher proportion of ACPs completed at baseline on the pilot ward $(38 \%)$ than in the retrospective review $(2 \%)$. The retrospective data reviewed all hospital deaths, and the pilot data was collected from a designated geriatrics ward where staff are more likely to be familiar with ACP.

The three main interventions were:

> identifying appropriate patients using the ACP column on the multidisciplinary board round

$>$ doctor education and ACP champions

$>$ improved communication with primary care.

The ACP column served as a daily reminder to prioritise these discussions.

Education was key to giving confidence to doctors to initiate conversations. The skills learned are transferable to other specialties.

The support of senior management is key to the sustainability of improvement projects. ${ }^{26}$ The ACP project was strongly supported by the departmental lead and senior doctors within the geriatrics department. The role of the ACP champions was essential, highlighted by the drop in ACPs when they were absent. They prompted daily ACP discussion of patients until the practice was embedded. To ensure sustainability, ACP champions were also tasked with handing over the role to their replacement colleague when they rotated.

We hope that the QIP has laid the foundations for an established change in practice using the multidisciplinary board rounds and a skilled workforce who are educated in initiating, documenting and communicating ACP discussions.

In the pre-project audit, patients spent on average 16.9 days in hospital before dying. In the follow up study, 18 out of 24 patients who were discharged avoided readmission. If these patients had been readmitted and died in hospital, it is assumed they would have also spent approximately 16.9 days each in hospital, equating to 304 bed days. The Department of Health and Social Care estimates that one patient bed day costs $£ 400{ }^{32}$ If these 18 patients had been readmitted and died in hospital, this would have cost an estimated $£ 121,000$. This figure represents the potential saving for only one ward over 4 months.

End-of-life care costs are significant in the community. However, these have been shown overall to be cheaper than hospital. ${ }^{10}$

In the pilot study, 44 patients had an ACP but 20 died before discharge indicating that some ACP discussions needed to be initiated earlier.

\section{Limitations}

In the pre-project audit, it was not possible to retrieve 30 casenotes from the 80 deaths recorded. However, the notes reviewed were assumed to be representative of the total cohort. 
When deciding if a patient was appropriate for an ACP, the teams discussed this between themselves based on the advised triggers. This was not formally standardised. The data collection relied on the teams accurately recording if the discussions took place. Variability in what was viewed as 'initiating an ACP discussion' may have limited the project.

Discharge summaries were reviewed to establish if ACP discussions were documented. The ward notes were not reviewed to confirm whether a telephone conversation between the ward team and the GP had occurred. Consequently, it is possible that discrepancies exist between the ACP content discussed on the ward and what was communicated to the GPs.

Data on the outcomes of patients for whom ACP was appropriate and not yet initiated were not collected. Thus, the true impact of the QIP on hospital readmissions is unknown.

\section{Conclusions}

This quality improvement project demonstrates a simple and successful strategy for improving ACP in the hospital setting. The interventions have shown a sustained change in practice although it is essential that education must be ongoing.

The project could be applied to many hospital departments or community settings.

If these strategies are employed across primary and secondary care, more patients would be able to express their preferences regarding goals of treatment and so receive individualised care.

Suggested next steps include establishing mandatory training in ACP communication skills for all HCPs and training for online systems for communicating information, such as 'Coordinate My Care'.

Through local campaigns, patients should be encouraged to ask HCPs to have these discussions and feel empowered to take control of the remainder of their lives.

In summary, the simple interventions used in this project increased ACP within the secondary care setting, embedded it within geriatric ward practice and ensured more patients were provided with the opportunity to have their preferences at the end of life achieved.

\section{Supplementary material}

Additional supplementary material may be found in the online version of this article at www.rcpjournals.org/clinmedicine: S1 - Timeline.

\section{Acknowledgements}

The authors would like to thank Dr Lucy Owen, Dr Joanna Pleming, Dr Tamar Ish-Horowicz, Dr Areej Benafif, Dr Annie Pervaiz, Dr Suresh Sivarajah and Dr Naomi Newman.

\section{References}

1 The Gold Standards Framework. Advance care planning. GSF, 2018. www.goldstandardsframework.org.uk/advance-care-planning

2 Royal College of Physicians. Talking about dying: How to begin honest conversations about what lies ahead. London: RCP, 2018. www.rcplondon.ac.uk/projects/outputs/talking-about-dyinghow-begin-honest-conversations-about-what-lies-ahead

3 Seymour J, Horne G. Advance care planning for the end of life: an overview. In: Thomas K, Lobo B (eds), Advance care planning in end of life care. Oxford University Press, 2011:16-27.
4 Schickendanz AD, Schillinger D, Landefeld CS et al. A clinical framework for improving the advance care planning process: start with patients' self identified barriers. J Am Geriatric Society 2009;57:31-9.

5 Amblàs-Novellas J, Murray SA, Espaulella J et al. Identifying patients with advanced chronic conditions for a progressive palliative care approach: a cross-sectional study of prognostic indicators related to end-of-life trajectories. BMJ Open 2016;6:e012340.

6 Dying Matters. www.dyingmatters.org

7 Forma L, Rissanen P, Aaltonen M, Raitanen J, Jylha M. Age and closeness of death as determinants of health and social care utilization: a case-control study. Eur J Public Health 2009;19:313-8.

8 Pot AM, Portrait F, Visser G et al. Utilization of acute and long-term care in the last year of life: comparison with survivors in a population-based study. BMC Health Serv Res 2009;9:139.

9 Van den Block L, Deschepper R, Bilsen J, Van Casteren V, Deliens $L$. Transitions between care settings at the end of life in Belgium. JAMA 2007;298:1638-9.

10 Kernick LA, Hogg KJ, Millerick Y et al. Does advance care planning in action to usual care reduce hospitalisation for patients with advanced heart failure: A systematic review and narrative synthesis. Palliat Med 2018;32:1539-51.

11 Davidson SN. End-of-life care preferences and needs: Perceptions of patients with chronic kidney disease. Clin J Am Soc Nephrol 2010:5:195-204.

12 Tierney WM, Dexter PR, Gramelspacher GP et al. The effect of discussions about advance directives on patients' satisfaction with primary care. J Gen Intern Med 2001;16:32-40.

13 Barnes K, Jones L, Tookman A. Acceptability of an advance care planning interview schedule: a focus group study. Palliat Med 2007:21:23-8.

14 Wright AA, Zhang B, Ray A et al. Associations between end of life discussions, patient mental health, medical care near death, and caregiver bereavement adjustment. JAMA 2008;300:1665-73.

15 Simon J, Porterfield P, Bouchal SR, Heyland D. 'Not yet' and 'Just ask': barriers and facilitators to advance care planning - a qualitative descriptive study of the perspectives of seriously ill, older patients and their families. BMJ Support Palliat Care 2015;5:54-62.

16 Davison SN, Simpson C. Hope and advance care planning in patients with end stage renal disease: qualitative interview study. BMJ 2006;333:886.

17 Yates P. Advance care planning: The pros and cons. J Thorac Oncol 2017:12:S1642-3.

18 Danis M, Gerrity MS, Southerland LI et al. A comparison of patient, family and physician assessments of the value of medical intensive care. Crit Care Med 1988;16:594-600.

19 Abadir PM, Finucane TE, McNabney MK. When doctors and daughters disagree: twenty-two days and two blinks of an eye. J Am Geriatr Soc 2011;59:2337-40.

20 Detering KM, Hancock AD, Reade MC et al. The impact of advance care planning on end of life care in elderly patients: Randomised controlled trial. BMJ 2010;340:c1345.

21 Scott IA, Mitchell GK, Reymond EJ, Daly MP. Difficult but necessary conversations - the case for advance care planning. Med J Aust 2013;199:662-6.

22 Prod'homme C, Jacquemin D, Touzet $L$ et al. Barriers to end-of-life discussions among hematologists: A qualitative study. Palliat Med 2018;32:1021-9.

23 Mason B, Sives D, Murray S. Advance care planning in the community. In: Thomas K, Lobo B (eds), Advance care planning in end of life care. Oxford University Press, 2011:148-57.

24 Brighton LJ, Bristow K. Communication in palliative care: talking about the end of life, before the end of life. Postgrad Med J 2016;92:466-70.

25 Almack K, Cox K, Moghaddam N, Pollock K, Seymour J. After you: conversations between patients and healthcare professionals in planning for end of life care. BMC Palliat Care 2012;11:15. 
26 Dixon J, Knapp M. Whose job? The staffing of advance care planning support in twelve international healthcare organizations: a qualitative interview study. BMC Palliat Care 2018;17:78.

27 Llewellyn H, Neerkin J, Thorne L et al. Social and structural conditions for the avoidance of advance care planning in neurooncology: a qualitative study. BMJ Open 2018;8:e019057.

28 Weiner JS, Cole SA. Three principles to improve clinician communication for advance care planning: Overcoming emotional, cognitive, and skill barriers. J Palliat Med 2004;7:817-29.

29 Wuensch A, Goelz T, Ihorst G et al Effect of individualised communication skills training on physicians' discussion on clinical trials in oncology: results from a randomised controlled trial. BMC Cancer 2017:17:264

30 Mudge AM, Douglas C, Sansome X et al. Risk of 12-month mortality among hospital inpatients using the surprise question and SPICT criteria: a prospective study. BMJ Support Palliat Care 2018:8:213-20.

31 NHS. Five year forward view. NHS, 2014. www.england.nhs.uk/wpcontent/uploads/2014/10/5yfv-web.pdf

32 Department of Health and Social Care. End of life care strategy: Promoting high quality care for all adults at the end of life. DoH, 2008. www.gov.uk/government/publications/end-of-life-carestrategy-promoting-high-quality-care-for-adults-at-the-end-of-theirlife

Address for correspondence: Dr Anna Steel, Department of Geriatrics, Barnet Hospital, Wellhouse Lane,

Barnet EN5 3DJ, UK.

Email: annasteel@nhs.net

\section{Royal College} of Physicians

\section{Acute care toolkit 15: Managing acute care problems in pregnancy}

Pregnant women can present to any acute hospital service at any time during their pregnancy or the postpartum period, up to 12 months post-delivery. Women may present with acute medical problems that need to be managed differently because of pregnancy, or may present with obstetric syndromes.

This toolkit provides practical guidance on managing women with acute medical problems in pregnancy. It also advises healthcare staff to take the opportunity to ask about a woman's mental wellbeing when she comes to hospital for other reasons during or after pregnancy; women with a history of mental health problems are more likely to develop new symptoms during pregnancy and postpartum.

Essential reading for front-line NHS staff who may be unfamiliar with the normal physiology of pregnancy and/or diseases that present in pregnancy.

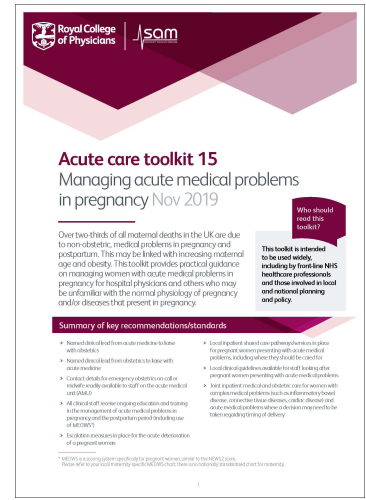

Download now at: www.rcplondon.ac.uk/act15 